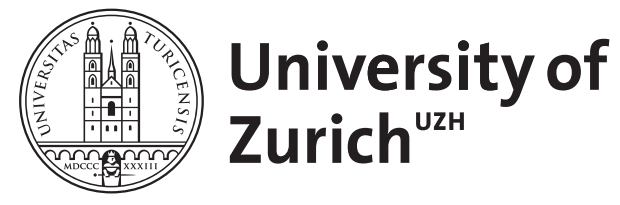
Archive

University of Zurich

University Library

Strickhofstrasse 39

CH-8057 Zurich

www.zora.uzh.ch

Year: 2011

Orbito-nasal cyst in a young European short-haired cat

Zemljič, T ; Matheis, F L ; Venzin, C ; Makara, M ; Grest, Paula ; Spiess, Bernhard M ; Pot, Simon A

DOI: https://doi.org/10.1111/j.1463-5224.2011.00930.x

Posted at the Zurich Open Repository and Archive, University of Zurich

ZORA URL: https://doi.org/10.5167/uzh-49759

Journal Article

Accepted Version

Originally published at:

Zemljič, T; Matheis, F L; Venzin, C; Makara, M; Grest, Paula; Spiess, Bernhard M; Pot, Simon A (2011). Orbito-nasal cyst in a young European short-haired cat. Veterinary Ophthalmology, 14(S1):122-129.

DOI: https://doi.org/10.1111/j.1463-5224.2011.00930.x 


\section{Orbito-nasal cyst in a young European short-haired cat}

2 Tadej Zemljič, DVM, Franziska L. Matheis, DVM, Dr. med. vet., "Claudio Venzin, DVM, 3 Dr. med. vet., Dipl. ECVS, $\dagger$ Mariano Makara, DVM, Dipl. ECVDI, $\$$ Paula Grest, DVM, Dr.

4 med. vet., Dipl. ECVP,§ Bernhard M. Spiess, DVM, Dr. med. vet., Dipl. ACVO/ECVO* and 5 Simon A. Pot, DVM, Dipl. ACVO/ECVO"

6 "Equine Department, Section of Ophthalmology, Vetsuisse Faculty, University of Zurich, CH-

78057 Zurich, Switzerland, † Department of Surgery, Vetsuisse Faculty, University of Zurich,

8 CH-8057 Zurich, Switzerland, $\$$ Department of Diagnostic Imaging, Vetsuisse Faculty,

9 University of Zurich, CH-8057 Zurich, Switzerland, § Institute of Veterinary Pathology,

10 Vetsuisse Faculty, University of Zurich, CH-8057 Zurich, Switzerland

Address communications to:

Tadej Zemljič

Klinika za male živali

Gorkičeva 6

SI-1000 Ljubljana

Slovenia

Tel.: +386 31550520

Fax: +38614235024

E-mail: zemljita@yahoo.com 


\section{ABSTRACT}

Purpose To describe a case of an orbito-nasal cyst in a cat.

Procedure An eighteen months old male European short-haired cat was presented to the Ophthalmology service of the Vetsuisse Faculty, University of Zurich for a subcutaneous swelling in the medial canthal region of the right eye (OD). Ophthalmologic, ultrasound and CT examinations, and fine needle aspiration were performed. After lesion excision the removed tissue was submitted for histopathology. CT examination was repeated 5 months after removal of the cyst.

Results Ophthalmologic examination revealed a large fluctuant swelling inferonasal to OD. Despite patent lacrimal puncta, only the first few $\mathrm{mm}$ of the lacrimal canaliculi could be cannulated. A normal globe with moderate enophthalmos was present. Ultrasound examination showed a well-defined lobulated cyst-like structure in the right orbit, inferonasal and anterior to the eye. CT examination revealed extension of this lesion through the medial orbital wall into the right nasal cavity. Fine needle aspiration confirmed the cystic nature of the lesion. An orbito-nasal cyst was diagnosed. The orbital part of the cyst was dissected from the surrounding tissue and excised from the periosteum in the medial orbital wall defect. Part of the maxillary bone was removed to allow removal of the cyst from the nasal cavity. Histologically, the cyst wall consisted of a single to multi-layered, mostly cuboidal epithelium and surrounding connective tissue. Follow-up revealed a good functional result and no recurrence 7 months after cyst removal.

Conclusions Similar orbito-nasal cystic structures were reported in dogs, but not in cats.

Key Words: orbito-nasal cyst, cat, CT, NL duct, excision, histopathology 


\section{INTRODUCTION}

Cystic lesions in the periorbital area are rare in domestic animals.(1) In dogs, reported cases include dacryops, $(1,2,3,4)$ congenital medial canthal cyst,(5) canaliculops,(6) cystic dilatation of the nasolacrimal $(\mathrm{NL})$ duct, $(7,8)$ maxillary bone epithelial cyst, $(9,10)$ suspected epithelial cyst,(11) periorbital epidermoid cyst,(12) cholesterol granuloma,(13) zygomatic salivary mucocele and cysts of the frontal and nasal sinuses.(1)

A dacryops is a cyst originating from lacrimal glandular ductal tissue, which can be confirmed histologically. $(2,3)$ Specifically, myoepithelial cells, which are present in tissues around lacrimal gland acini and ductules, can be detected via immunohistochemical staining for smooth-muscle actin. This was recently described in a report of 3 cases of dacryops in young Labrador retrievers by Ota et al.(2)

This case report describes the clinical presentation, diagnosis, surgical treatment and postoperative follow-up of an orbito-nasal cyst in a cat.

\section{CASE REPORT}

\section{Case history}

An 18 months old, intact male, domestic shorthaired cat was presented to the Ophthalmology service of the Vetsuisse Faculty, University of Zurich, Switzerland, for the evaluation of a subcutaneous swelling in the medial canthal area of the right eye. The cat belonged to the Institute for Animal Nutrition at the Vetsuisse Faculty of Zurich, and was engaged in a nutritional study. The exact duration of this swelling was unknown, but mild swelling had already been noticed when the cat was a kitten, and had started to enlarge over the last several months. According to the caretaker, the swelling was not associated with any discomfort but the eye had frequently been tearing since the cat was a few months old. There was no known history of trauma. 
Previous problems associated with this eye include conjunctivitis, keratitis and lower eyelid entropion. A PCR for detection of FHV-1 DNA from a cytobrush sample taken from the lower conjunctival fornix had been performed previously at a time when the cat also demonstrated signs of rhinitis. Although the result was negative, FHV-1 was suspected as the etiologic agent for the conjunctival and corneal inflammation. Also, the lower eyelid entropion was suspected to be the result of longstanding blepharospasm, due to painful herpetic ocular surface disease. Since entropion did not resolve after temporary tarsorrhaphy, permanent correction with a Hotz-Celsus procedure was performed, which restored a normal lower eyelid position.

\section{Clinical findings}

The results of a neuro-ophthalmological examination, including menace response, dazzle, pupillary and palpebral reflexes were normal. A well-circumscribed, oval fluctuant subcutaneous swelling was present inferonasal to the right eye. (Fig 1) The swelling was nonmovable, was not warm or painful, but did appear tender on firm palpation. There was some dried mucoid discharge on the eyelids, especially in the medial canthus, but the skin overlying the swelling was unremarkable. No obvious epiphora was present. The eyelid fissure was narrower compared to that of the other eye. Motility of the lower eyelid was impaired due to the swelling, but the eyelid fissure could still be closed completely. The right globe was moderately enophthalmic, and obvious resistance was felt on retropulsion in a ventral and medial direction. Retropulsion in other directions was unremarkable. No pain was elicited with retropulsion or opening of the mouth.

Eyelid margin position was appropriate, but there was some fibrosis of the palpebral conjunctiva in the medial canthus, including the area of the lower lacrimal punctum. With the lower eyelid in an everted position, the swelling could also be appreciated beneath the nasal palpebral conjunctiva. 
Passage of fluorescein dye through the nasolacrimal duct (Jones test I) was absent OD. The lacrimal puncta OD could be cannulated, but the cannula could only be advanced for one to two mm into the lacrimal canaliculi. The NL system could therefore not be irrigated.

The palpebral conjunctiva was mildly hyperemic. Mild fibrosis and fine ghost vessels were present in the inferotemporal cornea, but no retainment of fluorescein stain was observed.

The rest of the ophthalmic examination of the right eye, including slit-lamp biomicroscopy, indirect ophthalmoscopy and applanation tonometry, did not reveal any abnormalities. The examination of the left eye was unremarkable. Beside mild mucopurulent discharge around the right nostril, the physical exam was unremarkable.

\section{Preoperative imaging}

On ultrasound examination of the right orbital area, a lobulated structure with a well-defined contour and anechoic content was observed in contact with the inferonasal aspect of the right globe. The morphology and content of this structure was consistent with a cyst. The right globe was unremarkable. (Fig 2)

Upon aspiration of the cyst, a slightly opaque, brownish fluid was obtained. The cyst fluid had a specific gravity of 1.006 , protein content of $<1 \mathrm{~g} / 1$ and cell count of $13 / \mu 1$. Cytological evaluation of the cyst fluid revealed a moderate number of erythrocytes and few neutrophills and small lymphocytes.

Based on the results of the clinical examination, orbital ultrasound and analysis of aspirated fluid, an orbital cyst was diagnosed.

In order to evaluate the extension and possible associated bone involvement, a CT scan of the head was performed with the animal under anesthesia. The CT study was done using a multirow unit ${ }^{\#}$, with a beam collimator of 40 data channels with a detector-row width of 0.6 millimeters ( $40 \times 0.6 \mathrm{~mm}$ ), a tube potential of 120 kilovolts (peak kilovoltage) and a tube

\footnotetext{
\# Somatom Sensation Open, Siemens AG, Medical Solutions, Erlangen, Germany
} 
current of 200 milliamperes. Images were reconstructed with a section thickness of $0.75 \mathrm{~mm}$, using both a soft tissue and a bone window. (Fig 3) A post contrast scan was obtained 30 seconds after the intravenous injection of an iodinated high osmolar contrast medium ${ }^{* *}$ with a dose of $700 \mathrm{mgI} / \mathrm{kg}$ and a concentration of $350 \mathrm{mgI} / \mathrm{ml}$. The CT images revealed a severe enlargement of the osseous component of the lacrimal canal. Additionally, an ovoid structure with soft tissue density and well-defined contours extended from the ventromedial aspect of the eye, through the widened lacrimal canal and into the nasal cavity. This structure displaced the right eye caudoventrally and the ventral nasal conchal bone axially. Three-dimensional bone reconstructions are shown in Fig 4. For 3-dimensional reconstructions, the pixel values of the cyst have been changed to demonstrate the location and extension of the cyst. Attempts to identify and cannulate the nasal ostium of the NL duct were unsuccessful.

Because the lacrimal canaliculi could not be cannulated either, dacryocystorhinography could not be performed.

Since the cyst was expanding significantly, progressively dislocating the globe and obstructing the visual axis, and the cyst refilled within days after fluid aspiration, a surgery to remove the cyst was recommended and scheduled.

Surgery

The cat was premedicated with ketamine hydrochloride $(8 \mathrm{mg} / \mathrm{kg}$ of body weight (BW); Narketan ${ }^{\circledR}$, Vétoquinol) in combination with midazolam $\left(0.1 \mathrm{mg} / \mathrm{kg}\right.$ BW; Dormicum ${ }^{\circledR}$, Roche $)$ administered intramuscularly. Induction was then performed with propofol (5 mg/kg BW IV; Diprivan $^{\circledR}$, AstraZeneca). After intubation, the cat was maintained on isoflurane (Attane $\mathrm{T}^{\mathrm{TM}}$ Isoflurane, Provet) and oxygen. Amoxicillin (20 mg/kg BW; Clamoxyl ${ }^{\circledR}$, Pfizer) was administered intravenously. A presurgical clip was performed on the right side of the head and the surgical field was aseptically prepared. The cat was positioned in left lateral recumbency.

\footnotetext{
Telebrix ${ }^{\circledR}, 350 \mathrm{mgI} / \mathrm{ml}$, Guerbert, Zurich, Switzerland
} 
A $3 \mathrm{~cm}$ skin incision was performed $1.5 \mathrm{~cm}$ below and parallel to the lower eyelid margin.

The orbital part of the cyst was bluntly dissected from the surrounding tissues. (Fig 5) During dissection a fibrous strand of tissue, running from the dorsal cyst wall to the palpebral conjunctiva close to the lower nasal eyelid margin, was identified. A small perforation of the cyst wall, resulting in collapse of the cyst, occurred when the dissection approached the periosteum of the maxillary bone. To facilitate the rest of the dissection, the cyst was filled with $1.2 \%$ hyaluronic acid (Biovisc $1.2 \%{ }^{\circledR}$, Acrivet). The cyst was then excised at the level of the periosteum of the inferonasal orbital rim, revealing an oval defect (maximal diameter: 12 $\mathrm{mm}$ ) in the lacrimal and maxillary bone. (Fig 6) The extension of the cyst into the nasal cavity could be identified through this defect. Since the cyst in the nasal cavity expanded well beyond the margin of the bone defect, this opening needed to be enlarged in order to remove the cyst. This was accomplished in two steps. First, the compact bone was removed with use of a pneumatic bone drill. Then, the rest of the bone was removed with angled Cicherelli and Kerrison rongeurs. Cyst tissue was removed from the nasal cavity, enabling direct visualization of nasal turbinates. The cyst was placed in $10 \%$ neutral buffered formalin and

At the conclusion of surgery, meloxicam $\left(0.1 \mathrm{mg} / \mathrm{kg} \mathrm{BW}\right.$; Metacam ${ }^{\circledR}$, Boehringer Ingelheim $)$ and buprenorphine $\left(0.015 \mathrm{mg} / \mathrm{kg} \mathrm{BW}\right.$; Temgesic ${ }^{\circledR}$, Schering-Plough) were administered intravenously for postoperative analgesia. Recovery from anesthesia was uneventful. The cat was released from the hospital the same day, with the following therapy: amoxicilin / clavulanic acid (Clavubactin $^{\circledR}$, Dr. E. Graeub) $20 \mathrm{mg} / \mathrm{kg}$ BW BID PO for 14 days, meloxicam 
173 oral suspension for cats (Metacam ${ }^{\circledR}$, Boehringer Ingelheim) $0.1 \mathrm{mg} / \mathrm{kg} \mathrm{BW}$ once daily for the

174 first day after the surgery, followed by $0.05 \mathrm{mg} / \mathrm{kg} \mathrm{BW}$ once daily for 5 more days, $0.3 \%$

175 tobramycin eyedrops (Tobrex ${ }^{\circledR}$, Alcon) and lubricating gel with Vitamin A $\left(\right.$ Oculotect $^{\circledR}$,

176 Novartis) QID OD for 14 days. An Elizabethan collar was recommended for 14 days until

177 removal of the external sutures.

178 Histology

179 Examination of sections stained with haematoxylin and eosin (HE) demonstrated a cyst wall 180 consisting of a single or double and rarely multiple layers of mostly cuboidal epithelial cells 181 forming a stratified epithelium surrounded by a highly cellular connective tissue capsule. No 182 goblet cells or cilia could be detected. Multiple deposits of well-mineralized metaplastic bone 183 and lymphocytic aggregates were present in the surrounding connective tissue. (Fig 7) Immunohistological staining for smooth-muscle actin was performed and was found negative (data not shown). The histological diagnosis was a non neoplastic cyst.

\section{Follow up}

The wound had healed nicely on recheck three weeks after surgery. (Fig 8) Mild enophthalmus and nictitating membrane protrusion were present and the palpebral fissure was narrower compared to that of the other eye. The palpebral reflexes were normal, although the motility of the lower lid was still mildy impaired. No difference in retropulsion was present between both eyes. Also, no pain was elicited with palpation of the eyelids and retropulsion of the globe. No epiphora was present, but some dried, mucoid discharge was present around the right nostril. No stridor was detected. No other changes from previous examinations were observed in either eye. No further therapy was prescribed.

195 On recheck 5 months after the cyst removal, the right palpebral fissure was mildly narrowed and mild epiphora from the right eye was present. There was mild entropion without trichiasis 
of the lower eyelid margin on the operated side, and no discomfort was noticed by the examiners, nor reported by the handler. The rest of the ocular exam was unremarkable in both eyes.

\section{Postoperative imaging}

In the same follow-up examination, 5 months after the surgery, the cat was anaesthetized as previously described, and the CT examination was repeated. The previously identified cystlike structure could not be identified. (Fig 9) There was a persistent mild caudal and ventral displacement of the right eye. The widened osseous component of the lacrimal canal was unchanged compared to the pre-operative study. The right ventral conchal bone presented a normal position and the left ventral nasal concha was filled by a soft tissue dense material. This finding was interpreted as rhinitis. Additionally, a defect on the tip of the root of the upper right canine tooth was identified. This lesion was likely the consequence of iatrogenic trauma during maxillary bone removal.

The following options were given by the Dentistry service: recheck with dental radiographs in 4 months, apical preparation with retrograde filling of the root canal or extraction of the tooth. The prognosis of the retrograde filling procedure would be guarded at best due to various reasons. The handler has opted for observation and regular rechecks, and would decide for an extraction of the tooth in case of any problems.

On the last recheck, 7 months after surgery, no changes from the previous exam were noticed. No pain or ocular discomfort was reported by the caretaker, but constant mild tearing from this eye was present. (Fig 10)

\section{DISCUSSION}

Periorbital cysts are rare in domestic animals,(1) and may originate from several glandular or ductular structures, including the orbital lacrimal gland, lacrimal gland of the nictitating 
membrane, zygomatic salivary gland, lacrimal canaliculi, sac and/or NL duct, conjunctival goblet cells, frontal and nasal sinus mucosa, and transposed parotid duct.(1,6) The etiology of these cysts is various, and includes congenital malformation, trauma, inflammation and neoplasia.(1) In the report by Martin et al., the anatomic location of the cyst and any associated lesions was stated to be the most consistent clue to its origin.(1)

This report describes the case of an orbito-nasal cyst in a young cat. The origin of the cyst, as well as its etiology, remains unknown. Due to the anatomic location, it is very likely that the cyst was closely associated with, or originated from, the lower lacrimal canaliculus or nasolacrimal duct.

Although we could not find any reports of orbito-nasal cysts in cats, there are reports describing similar conditions in dogs.

A case of a nasal and orbital lacrimal cyst, accompanied by occlusion and dilatation of the NL duct, was described by Grahn et al. in a six months old basset hound.(3) White et al. described a case of a cystic obstruction of the nasolacrimal duct in a one year old dachshund.(7) Ito et al. reported a cyst, suspected to be epithelial, that occupied a defect in the maxillary, lacrimal and frontal bones and invaded the nasal cavity in a 4-year old miniature schnauzer.(11)

Although the clinical presentation in these cases was similar in that cystic structures affecting the orbit and the nasal cavity with a defect in the orbital wall were present, there were some differences regarding the tissue of origin and possible etiology of the cyst. In the report by Grahn et al., the seromucinous glandular tissue identified in the cyst wall most likely originated from the third eyelid gland, and a congenital lesion was suspected. The NL duct was occluded by the cyst, and dilatation of the duct might have been related to continuous tear flow.(3) In the case described by White et al., the cyst consisted of the distended NL duct due 
to distal obstruction by a chronic inflammatory process. The specific cause for the inflammation was not identified.(7) In the report by Ito et al., the etiology was unknown, and although no abnormalities of the NL duct were reported, the duct was still thought to be the most likely structure of origin.(11)

Dacryocystorhinography is a radiographic contrast examination procedure used to delineate the nasolacrimal drainage system and evaluate its patency.(14-16)

Since it was not possible to cannulate the lacrimal canaliculi or the nasal ostium of the NL duct, dacryocystorhinography could not be performed in this case. Therefore, eventual communication of the lumen of the cyst with the lumen of the NL system could not be ascertained. But even if dacryocystorhinography could have been performed, and the dacryocystorhinogram would not have identified a communication, a connection of the cyst wall with the NL duct could not have been ruled out. During surgery, a fibrous strand of tissue was found connecting the dorsal cyst wall and the palpebral conjunctiva close to the lower medial eyelid margin corresponding to the site of the lower lacrimal punctum. Although we cannot support this histopathologically, we think that this structure was most likely a fibrosed lower lacrimal canaliculus, and that the cyst was actually connected with the NL system.

Based on negative immunohistochemical staining for $\alpha$-smooth actin, we conclude that the cyst did not originate from lacrimal gland tissue or ductules,(2) which supports our assumption of a canalicular origin for the cyst.

To the best of our knowledge, there is no information in the literature regarding the histology of the NL duct in cats. In the dog, a bistratified columnar anatomy has been reported for the epithelium of the lacrimal sac and the proximal and middle portion of the nasolacrimal duct.(17) Identification of a single to multilayered, mostly cuboidal epithelium lining the cyst in our case, does not exclude the possibility that the cyst did originate from the NL system. 
Also, this finding makes it very unlikely that this was an epidermoid or dermoid cyst, both of which are characterized by a stratified squamous epithelium, or a cholesterol granuloma which lacks the epithelial layer. $(9,12,13)$

The defect in the orbital wall could be explained by bone atrophy due to pressure exerted by the enlarging cyst as was described previously. $(7,9)$

The nasal conchae, which were displaced by the cyst on preoperative CT, were found to be reorganizing on the postoperative $\mathrm{CT}$.

We hypothesize that the previously diagnosed conjunctivitis, keratitis and entropion were caused by FHV-1. The fibrotic changes of the palpebral conjunctiva and lacrimal canaliculi were interpreted as symblepharon, which is a well known sequel of FHV-1 induced ulcerative conjunctivitis.(18)

Despite the negative result of a PCR assay for the detection of FHV-1 DNA, FHV-1 should not necessarily be ruled out as the etiologic agent for the above mentioned changes.(19) Many cats which are likely to have FHV-1 associated disease display negative PCR results.(20)

It is possible that viral DNA was not present in the tested sample or was not present in detectable amounts.(19, 21) Alternatively, the cat might not have been shedding virus at the time of sampling.(19)

It is impossible to say whether the nasal ostium of the NL duct was actually absent or we were just unable to localize it.

Removal of the cyst was advised and performed because the increasing size of the cyst was causing globe displacement and obscuration of the visual axis. Also, the cyst refilled quickly after aspiration of the fluid and further enlargement of the cyst was therefore expected. Possible treatment alternatives have been reported in the literature. The intralesional 
application of tetracycline has been mentioned as a fibrosing agent for the treatment of periocular cysts, but at the same time was not recommended by the author because of possible conjunctival necrosis and severe periocular inflammation.(22) Sclerotherapy with ethanolamine oleate was recently described for the management of an orbito-palpebral cyst associated with congenital microphthalmos of a non-visual eye.(23) The use of polidocanol, another sclerosing agent, has been reported anecdotally as therapy for dacryops in dogs, but no reports have been published to this date. Due to the size and extension of the cyst, the possible side effects of a treatment with sclerosing agents and the lack of publications supporting the use of such a therapy, chemical ablation of the cyst was not attempted in this case and surgical excision was performed instead.

No recurrence of the cyst was noticed on repeat CT examination 5 months after surgery. Mild enophthalmos, a narrowed palpebral fissure, epiphora, mild inversion of the lower eyelid margin and nictitating membrane protrusion were noticed on rechecks 5 and 7 months after cyst removal. The presence of enophthalmos, nictitating membrane protrusion and an abnormal eyelid position might be attributed to the osseous defect in the medial orbital wall and/or atrophy of the orbital fat due to the pressure exerted by the enlarging cyst. No discrepancy in the size of the right and left orbit or globe was noticed on the CT images. Protrusion of the nictitating membrane, both before and after surgery, could also be attributed to symblepharon, although no obvious restriction of nictitating membrane motility was noticed during or after cyst removal. Epiphora was thought to be caused by a combination of absent tear drainage through the nasolacrimal system and presence of mild tipping in of the lower eyelid margin. Since no trichiasis was present and no discomfort was noticed at recheck examinations nor reported by the handler, correction of this mild entropion was judged to be unnecessary. The injury of the root of the upper canine tooth that occurred during the removal of bone was accidental, and was not noticed intraoperatively. Considering the anatomical location of the tooth, it is possible that trauma to the tooth root occurred unnoticed, and this 
emphasizes the importance of extreme caution during removal of bone in such proximity to the teeth.

Since this is an intact male cat, which at least occasionally had contact with other cats, ocular trauma cannot be ruled out completely as contributing factor in the cyst formation. Also, considering the young age of the cat, a congenital abnormality is possible.

In conclusion, the presentation, diagnosis, removal and long-term follow-up of an orbito-nasal cyst are described. The origin and etiology of the cyst remain unknown. Removal of the cyst resulted in a good functional outcome and lack of recurrence 7 months after surgery.

\section{REFERENCES:}

1. Martin CL, Kaswan RL, Doran CC. Cystic lesions of the periorbital region. Compendium on Continuing Education for the Practicing Veterinarian 1987; 9: 1022-1027.

2. Ota J, Pearce JW, Finn MJ at al. Dacryops (lacrimal cyst) in three young Labrador retrievers. Journal of the American Animal Hospital Association 2009; 45: 191-196.

3. Grahn BH, Mason RA. Epiphora associated with dacryops in a dog. Journal of the American Animal Hospital Association 1995; 31: 15-19.

4. Playter RF, Adams LG. Lacrimal cyst (dacryops) in 2 dogs. Journal of the American Veterinary Medical Association 1977; 171: 736-737.

5. Cullen CL, Grahn BH. Diagnostic ophthalmology. Congenital medial canthal cyst. The Canadian Veterinary Journal 2003; 44: 935-937.

6. Gerding PA. Epiphora associated with canaliculops in a dog. Journal of the American Animal Hospital Association 1991; 27: 424-426. 
7. White RAS, Herrtage ME, Watkins SB. Endoscopic management of cystic naso-lacrimal obstruction in a dog. Journal of Small Animal Practice 1984; 25: 729-735.

8. Lavach JD, Severin GE, Roberts SM. Dacryocystitis in dogs: a review of twenty-two cases. Journal of the American Animal Hospital Association 1984; 20: 463-467.

9. Featherstone H, Labrets Diaz F. Maxillary bone epithelial cyst in a dog. Journal of Small Animal Practice 2003; 44: 541-545.

10. Lin CT, Tasi WC, Hu CK et al. Maxillary bone epithelial cyst in adult miniature schnauzer. The Journal of Veterinary Medical Science 2008; 70: 1001-1004.

11. Ito K, Asano K, Urano T et al. Periorbital cyst with bone defect in dog. The Journal of Veterinary Medical Science 2006; 68: 747-748.

12. Davidson HJ, Blanchard GL. Periorbital epidermoid cyst in the medial canthus of three dogs. Journal of the American Veterinary Medical Association 1991; 198: 271-272. Practice 1990; 31: 208-211.

14. Barnett KC, Crispin SM. Lacrimal system. In: Feline Ophthalmology: An Atlas\&Text. Saunders, London, 1998; 61-68.

15. Yakely WL, Alexandra JE. Dacryocystorhinography in the dog. Journal of the American Veterinary Medical Association 1971; 159: 1417-1421.

16. Gelatt KN, Cure TH, Guffy MM, Jessen C. Dacryocystorhinography in the dog and cat. Journal of Small Animal Practice 1972; 13: 381-393. drainage system (abstract). European Veterinary Ophthalmology Meeting 2008: 57. 
362 18. Stiles J, Townsend WM. Feline ophthalmology. In: Veterinary ophthalmology 4th edition 363 (ed Gelatt KN). Blackwell Publishing: Ames, 2007; 1095-1164.

364 19. Maggs DJ. Feline herpesvirus : Diagnostic testing and management in multicat setting. 365 The Nordic Eye Meeting 2010.

366 20. Veir JK, Lappin MR. Molecular diagnostic assays for infectious diseases in cats.

367 Veterinary Clinics of North America: Small Animal Practice 2010; 40: 1189-1200.

368 21. Low HC, Powell CC, Veir JK et al. Prevalence of feline herpesvirus 1, Chlamydophila 369 felis, and Mycoplasma spp DNA in conjunctival cells collected from cats with and without 370 conjunctivitis. American Journal of the Veterinary Research 2007, 68: 643-648.

371 22. Giuliano EA, Moore CP. Diseases and surgery of the canine lacrimal secretory system. In:

372 Veterinary ophthalmology 4th edition (ed Gelatt KN). Blackwell Publishing: Ames, 2007; 373 633-661.

374 23. Naik MN, Murthy RK, Raizada K et al. Ethanolamine oleate sclerotherapy in the 375 management of orbito-palpebral cyst associated with congenital microphthalmos. American 376 Journal of Ophthalmology 2005; 139: 939-941. 